\begin{tabular}{lc}
\cline { 2 - 2 } International Journal of Engineering Sciences 2019 12(1) $24-33$ & INTERNATIONAL JOURNAL \\
OF \\
ENGINEERING SCIENCES \\
\hline
\end{tabular}

\title{
Correlation of Simulated Results with Experimental Modal Analysis for an Asymmetric Structure
}

\author{
Mitul Shah*, M. G. Karnik ${ }^{\$}$ \\ "M.Tech, Design Engineering, Mechanical Engg Dept., College of Engineering, Pune \\ ${ }^{\$}$ Associate Professor, Mechanical Engg Dept., College of Engineering, Pune \\ (Received 11 September 2018; accepted 14 March 2019) \\ https://doi.org/10.36224/ijes.120104
}

\begin{abstract}
The aim of this study is to investigate the reasons of the discrepancies in results of modal analysis due to Finite Element (FE) simulation and inaccuracies in Experimental Modal Analysis (EMA) for an asymmetric structure. An asymmetric structure with 87 stitch welds is simulated, tested and correlated. As the initial FE-EMA correlation showed frequency differences of over $24 \%$, an optimization model is developed for the model to bring FE results close to EMA. FE optimization is performed with each weld modeled as a separate component which led to FE-EMA frequency difference reduced from over $24 \%$ to about $10 \%$. The sources of error in FEA were identified as non-incorporation of manufacturing deviations such as efficacy of the welds, changes in stiffness due to changes in microstructure of Heat Affected Zone (HAZ), dimensional tolerances of components in assembly and variation of sheet thickness across the model.
\end{abstract}

Keywords: Asymmetric geometry, EMA, FE optimization, correlation, welded joints

\section{Introduction}

Sheet metal body is usually manufactured by bending the developed geometry followed by spot or stitch welds for assembly. However, for sheet structures with thickness less than 3 $\mathrm{mm}$, these welds could burn off the material introducing small discontinuities in the assembly at the joints. The thin rolled sheets also show non uniformity in the thickness along the length. A tolerance of $0.1 \mathrm{~mm}$ for a thin sheet of $1.2 \mathrm{~mm}$ represents variation of over $8 \%$ compared to $2 \%$ for a $5 \mathrm{~mm}$ thick sheet for the same tolerance. For sheet structures with thickness greater than $3 \mathrm{~mm}$, the discontinuities in assembly at the joints are less, as the material does not burn off during the welding. The change in the microstructure for thick sheet structure is comparatively less, as the thickness of welds at the HAZ is less than the thickness of the sheet.

$\mathrm{FE}$ is an idealistic numerical method which represents perfect joints, perfectly homogeneous material, no geometric irregularities and no change in material properties due to welds. But actual manufacturing constraints deviates the model from the ideal model. Therefore, thin sheet structures cannot be modeled as an integrated body in FE. In order to understand the effect of these geometric discontinuities on the FE-EMA correlation, an assembly of a thin sheet structure is dealt with in this study. In practical problems, symmetry may not be always possible even though it may be desired. Also, symmetric models are relatively easy to model as far as FE modelling is considered as they can be analysed only by considering a part of the model about symmetric planes. It is for this reason that an asymmetric model is manufactured for the study as entire model has to be modelled and there is no scope for eliminating parts or simplify model for analysis. The geometry considered is an asymmetric box structure with various components assembled together with 87 stitch welds. All the components are made from the $1.2 \mathrm{~mm}$ thick sheet of SS304. Slots on this model introduced variation in local stiffness. [1] discusses the various checks like modal effective mass to identify the flow of mass for a particular mode and use of strain energy to 
identify areas for change for proper interpretation of FE results in MSC. Nastran along with several other tools.

EMA is then performed on this asymmetric model by using roving accelerometer test for it gives a complete column of characteristic data. The test is performed with a soft hammer tip as the structure is a thin sheet structure [2]. Exponential and transient windows were used while performing the impact hammer tests. In his work, [1] has highlighted the importance of using multiple references so as not to skip acquisition of any mode. If a reference point lies on a node point (point of zero displacement) of a mode, then that mode will never be captured by the accelerometer. Hence, 5 reference points with an averaging of 3 hits per point is done in this experiment. [3] has highlighted various considerations while performing EMA and has discussed briefly about the SDOF and MDOF systems, different techniques of performing modal analysis along with coherence and post processing of EMA data.

Once FE and EMA data has been captured, correlation is performed between them. [4] proposed a new correlation improvement technique for the optimum node removal location to get improved modal assurance criterion (MAC) matrix. [5] performed EMA on a steel rod using PULSE vibration analyzer and found that with increasing frequency, MAC value decreases which is due to the fact that mode shape is more difficult with increasing frequency as the shape, which is measured experimentally, is dependent on density of defined degrees of freedom.

Although the highly sophisticated finite element method is used to predict dynamic behavior of assembled complete structures, the predicted results of assembled structures are often considerably different from the experimental results. The inaccuracy of predicted results is believed to be largely due to the invalid assumptions about the initial finite element models and/or initial parameter values. Therefore, model updating methods are usually used to improve the initial finite element models by using the measured results. [6] performed dynamic FE model update on the mounts designed for the engine of a 2 ton class Mini excavator using multi axial accelerations recorded using a data logger while the machine was in operation. [7][8] and [9] performed the sensitivity analysis considering Young's modulus, thickness or even offset and extensional offset parameters as variables whereas [10] has presented an integrated validation strategy that takes into account the complete process chain from model-based test design through modal testing, data evaluation, test/analysis correlation to computational model updating using ICS.sysval, a special MATLAB software package for model validation.[11] performed sensitivity analysis for a structure made of thin sheets (1.5 $\mathrm{mm}$ ) by using a combination of parameters like Young's modulus, shear modulus, Poisson's ratio, density, bend radius and thickness, and the properties of spot welds and their patches. The total error in FE model reduced by $33 \%$ after updating. Similar attempt is made in this work to optimize the FE model to take into account the manufacturing defects and discontinuity in the geometry.

\section{Experimental analysis}

\subsection{Selection of geometry}

Figure 1(a) and 1(b) illustrates the model under consideration. Initially, a symmetric trapezoidal box of overall dimension $512 \times 300 \times 152 \mathrm{~mm}$ and $1.2 \mathrm{~mm}$ thickness is made. The side plate 2 and 4 along with base plate is a single integrated structure referred to as base component. Side plates 1 and 3 were then stitch welded to this base component to form a symmetric trapezoidal box. Now, to modify this model for varying component stiffness, slots of different size and shapes were cut in the side plates 1 and 3 and the base component. The two cross members and the two brackets on side plate 4 were stitched welded to introduce localized change in the stiffness in the weld region on these components. Thus The long members were stitched welded on the base plate so as to increase its stiffness. Thus, the 
resulting model had no perfect symmetry about any plane and the components had varying stiffness. The total number of stitch welds in this model is 87 . The dimensions of the stitch weld were difficult to maintain uniformly throughout the structure as it was made of thin sheet of $1.2 \mathrm{~mm}$ thickness. As a result, TIG welding had to be performed with a low current so as to avoid burning of the sheet. Thus, because of the practical difficulties involved during welding of this model like thin sheet structure $(1.2 \mathrm{~mm})$, considerably small size of the model $(512 \times 300 \times 152 \mathrm{~mm})$ and no welding experience of such thin structures with the associated workshop, no weld drawings were followed in this exercise and were welded as per convenience of the welder.

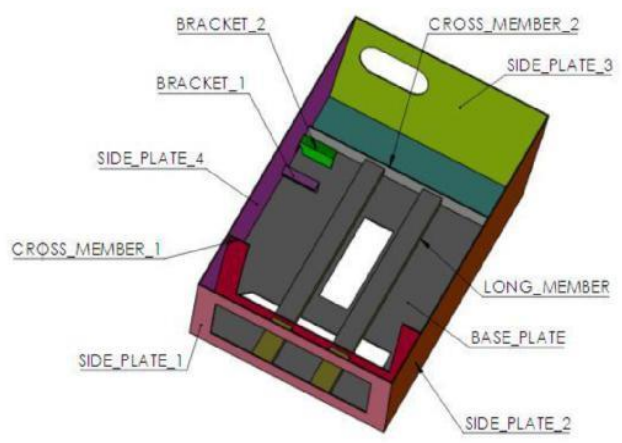

(a)

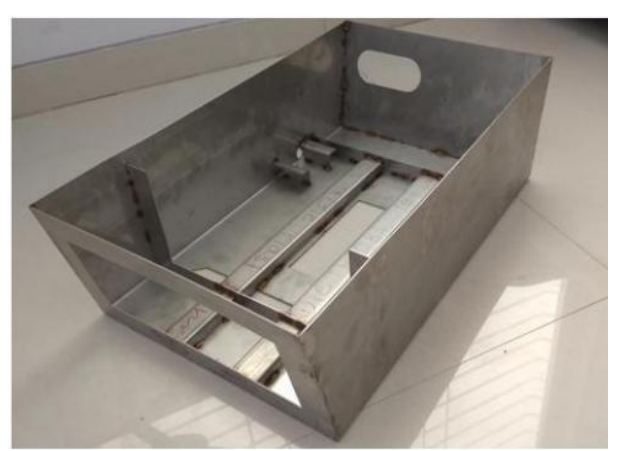

(b)

Figure 1: (a) Model in SolidWorks, (b) Manufactured model

\subsection{EMA setup and software used}

The EMA was performed with a 32 channel Brüel \& Kjær 3660-C-100 vibration analyzer. 6 tri-axial general purpose accelerometers of Brüel \& Kjær, 4535-B type with resonant frequency of $30 \mathrm{kHz}$ and weight of 6 grams each were used. A Brüel \& Kjær impulse hammer with sensitivity $2.27 \mathrm{mV} / \mathrm{N}$ was used with soft tip as the geometry was a thin structure. The software PULSE Labshop 21.0.0.567 was used for EMA and Altair HyperView/FEMtools3.7 was used for correlation.

\subsection{FE and EMA modeling}

Figure 2(a) shows the FE meshed model in HyperMesh. A solid model of this geometry is modeled in SolidWorks as shown in Figure 1(a) and was meshed by extracting mid-surface in Altair HyperMesh as it is a thin sheet structure. PSHELL properties were created for each component with the material SS304 and thickness of $1.2 \mathrm{~mm}$ assigned to it. The weld and noThe weld and no-weld regions were distinguished from each other as no weld region was represented with the free edges i.e. no equivalence at the interfaces. As the manufactured model was made without following specific weld drawings due to practical constraints like smaller size of the model, thin sheet structures, electric current regulating limitations in the TIG weld setup as discussed earlier, the span of each and every weld was modeled by measuring the corresponding lengths of each and every weld with the help of a ruler in the manufactured model. This method proves quite reliable as the size of the model is considerably small and number of welds is finite. However, for larger structures, it is recommended to follow the weld drawing and then incorporate welding deviation in the FE model if any. The total number of elements in this scheme is 45529 (190 CTRIA3 and 44339 CQUAD4). The CPU time for the run was 26 seconds with desktop PC with Intel Core i5 Processor (4x $2.5 \mathrm{GHz}$ ) and $6 \mathrm{~GB}$ DDR3 RAM with Optistruct used as a solver.

Figure 2(b) shows the corresponding EMA model in PULSE software. The actual manufactured model was marked with 84 points such that they were not only uniformly 
distributed on the surfaces but also enough spaced to perform EMA effectively and also to capture all the target modes. Reference point 1 is considered as origin and considering appropriate axes system, co-ordinates of all these 84 points were fed to the software to create the 3D grid of points which were connected to each other using 2D surface elements in the manner as in the actual model to create the geometry in PULSE software.

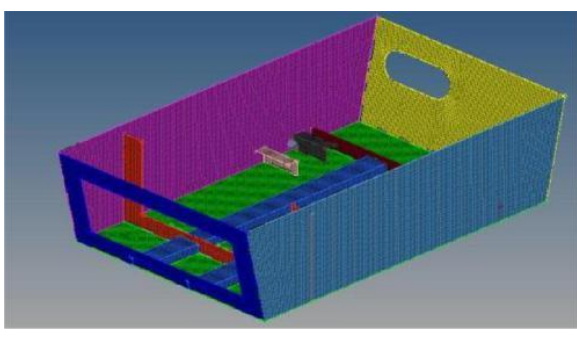

(a)

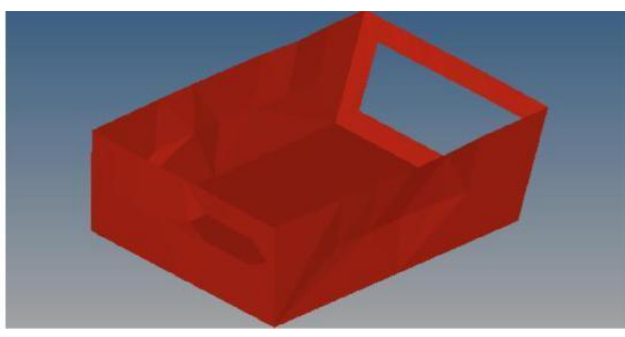

(b)

Figure 2: (a) FE model (b) EMA model in PULSE software

\subsection{Procedure followed to perform EMA}

Figure 3 illustrates the model hung horizontally about the corner points through 4 bungee cords to simulate free-free condition.



Figure 3: Experimental setup

Roving accelerometer technique was identified as a suitable technique to determine the natural frequencies and mode shapes. As 6 accelerometers were available; the readings were taken in 14 sets to cover all the 84 points.

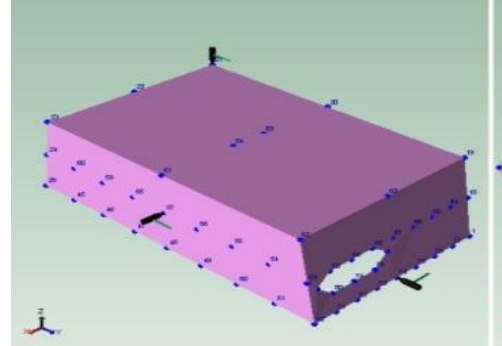

(a)

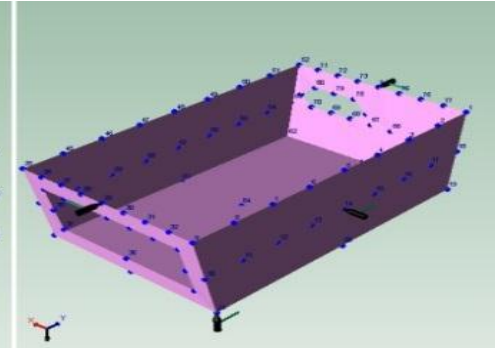

(b)

Figure 4(a), (b): EMA geometry and identification of hitting points

Figure 4 (a) and (b) highlights the 5 reference points (one reference per face) used in this experimentation. The selected impact points for this test were 14, 21, 29, 57 and 74. 
Transient window is used for force signal and exponential window is used for response as recommended for impact hammer test by [2]. The free-free test is then conducted taking an average of 3 readings per impact point. Thus, for a total of 84 accelerometer positions and 5 impact points, 1260 FRFs were obtained. These obtained FRFs are analyzed together using rational polynomial $-\mathrm{Z}$ curve fitter technique.

\section{Results and discussions}

Table 1 compares the first six flexible frequencies obtained by EMA with FE while table 2 shows the initial correlation. Initial comparison of the frequencies shows a maximum frequency difference of $24.28 \%$ for third mode and this difference further reducing to $0.44 \%$ for the higher mode at $125 \mathrm{~Hz}$.

Table 2: Initial MAC of the model with all

Table 1: Initial comparison 84 points

\begin{tabular}{|c|c|c|c|c|c|c|c|c|c|c|}
\hline $\begin{array}{l}\text { Mode } \\
\text { No. }\end{array}$ & $\begin{array}{l}\text { FEA } \\
(\mathrm{Hz})\end{array}$ & $\begin{array}{c}\text { EMA } \\
(\mathrm{Hz})\end{array}$ & $\begin{array}{c}\% \\
\text { Difference } \\
\end{array}$ & & $\begin{array}{c}\text { EMA } \\
1 \\
\end{array}$ & $\begin{array}{c}\text { EMA } \\
2 \\
\end{array}$ & $\begin{array}{c}\text { EMA } \\
3 \\
\end{array}$ & $\begin{array}{c}\text { EMA } \\
4 \\
\end{array}$ & $\begin{array}{c}\text { EMA } \\
5 \\
\end{array}$ & $\begin{array}{c}\text { EMA } \\
6 \\
\end{array}$ \\
\hline 1 & 56.81 & 53.73 & 5.42 & FEA 1 & 89.8 & 0.7 & 0.1 & 2.5 & 0.2 & 1.5 \\
\hline 2 & 61.52 & 69.45 & -12.9 & FEA 2 & 0 & 30.8 & 13.3 & 8.4 & 2.3 & 0.3 \\
\hline 3 & 64.75 & 80.47 & -24.28 & FEA 3 & 2.4 & 1.1 & 10.9 & 0.8 & 1.6 & 2.1 \\
\hline 4 & 73.4 & 83.28 & -13.46 & FEA 4 & 0.1 & 36.4 & 16.8 & 3.4 & 3 & 0.5 \\
\hline 5 & 77.05 & 93.61 & -21.49 & FEA 5 & 0.1 & 0.4 & 43.9 & 25.1 & 19.2 & 0.4 \\
\hline 6 & 124.36 & 124.91 & -0.44 & FEA 6 & 0.4 & 0.3 & 1 & 0 & 3.8 & 51.6 \\
\hline
\end{tabular}

These differences in frequencies can be explained by some assumptions, like: the numerical model built does not consider the manufacturing deviations such as efficacy of the welds, changes in material property due to changes in microstructure of HAZ, dimensional tolerance of components in assembly, geometric irregularities, stiffness variation etc. MAC is defined as the square of the cosine of the angle between the 2 normal at corresponding points in FE and EMA respectively. The poor value of MAC can be attributed to inaccurate capture of displacement which may be due to inaccurate capture of localized stiffness. Frequency is a function of mass and stiffness. If dynamic mass for a mode is inaccurately captured but the dynamic stiffness for that mode is accurately captured, then the frequency difference may be high but MAC too may be high as seen for mode 1 . Vice-versa case is observed for mode 6. Thus, the tangible reason for the poor MAC correlation was identified as reduced stiffness of some of the components in the manufactured model which could not be accurately modeled in FE. This conclusion was drawn by visually analyzing the simulations of the EMA in Altair Hyperview and confirming these observations by inspecting the model at these locations. Some points flex and showed large displacements due to reduced stiffness in these regions as shown in Figure 5 .



Figure 5: Large displacement and flex in EMA results 


\subsection{Optimization}

In order to bring the FE predictions close to that of the EMA, there was a need to perform optimization of the FE model on the material properties and variations in the sheet thickness. Hence, each and every part of the model along with the welds were modeled as separate components. Thus, there were a total of 95 components $(8$ model components and 87 weld components).

The range of the material properties selected for optimization is,

Young's Modulus - $180 \mathrm{GPa}$ to $205 \mathrm{GPa}$ for all components

$50 \mathrm{GPa}$ to $400 \mathrm{GPa}$ for weld components

Poisson's ratio -0.25 to 0.33 (ideal range for ductile materials)

Sheet thickness $-1.1 \mathrm{~mm}$ to $1.3 \mathrm{~mm}$

The band for the Young's modulus of the all components was kept from $180 \mathrm{GPa}$ to $205 \mathrm{GPa}$ as SS304 cannot have Young's modulus above $205 \mathrm{GPa}$, while for that of weld components, this band was kept from $50 \mathrm{GPa}$ to $400 \mathrm{GPa}$ with the initial value of Young's modulus set at $200 \mathrm{GPa}$. The reason for keeping such a wide band for the welds is because mechanical properties change in the weld zone. The Poisson's ratio was allowed to vary from 0.25 to 0.33 for all the components with the initial value kept at 0.29 . This is a realistic range for ductile materials. The thickness was allowed to vary from $1.1 \mathrm{~mm}$ to $1.3 \mathrm{~mm}$ with the initial value kept at $1.2 \mathrm{~mm}$. Actual verification of thickness at different locations confirmed this band.

Thus, an optimization model was made with a total of 285 design variables with an objective function to minimize the error in the frequencies as,

Minimize $(53.72-\mathbf{a})^{2}+(69.45-\mathbf{b})^{2}+(80.47-\mathbf{c})^{2}+(83.28-\mathbf{d})^{2}+(93.45-\mathbf{e})^{2}+(126.56-\mathbf{f})^{2}$

Subject to, $180<=\mathbf{E} m<=205$;

$$
\begin{aligned}
& 50<=\mathbf{E} w<=400 ; \\
& \mathbf{E} i=200 ; \\
& 0.25<=\mathbf{N u}<=0.33 ; \\
& \mathbf{N u} i=0.29 ; \\
& 1.1<=\mathbf{h}<=1.3 ; \\
& \mathbf{h} i=1.2
\end{aligned}
$$

where,

a-f are the first six FE frequencies,

$\mathbf{E} m$ is the Young's modulus of the all components,

$\mathbf{E} w$ is the Young's modulus of the weld components,

$\mathbf{E} i$ is the initial value of Young's modulus,

$\mathrm{Nu}$ is the Poisson's ratio,

$\mathrm{Nu} i$ is the initial value of the Poisson's ratio, 
$\mathbf{h}$ is the thickness of the sheet,

$\mathbf{h} i$ is the initial value of thickness

This model was then solved in Altair Optistruct (Design optimization). The value of the error function reduced from the initial 740.3 to 244.06 in 6 iterations as shown in the Figure 6. The updated property values from the optimization were then reassigned to the existing model and this updated model was solved for normal modes in Optistruct.

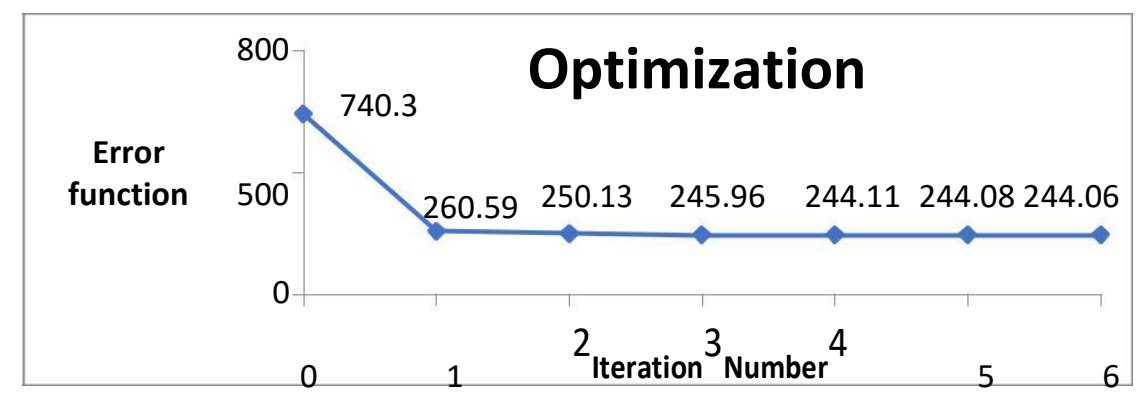

Figure 6: Optimization in Optistruct

The properties that were obtained after optimization showed varying values of Young's modulus for the weld components. While majority of the components attained value within $250 \mathrm{GPa}$, some of the components attained the Young's modulus of $400 \mathrm{GPa}$. These components were identified in the actual component. Visual inspection of the component showed that the bracket component was partially burnt at the top edge and had a large HAZ at the bottom edge. As the sheet is thin, the welds could have changed the microstructure of the material in these regions and made it stiff. Hence, the FE optimization prompted higher stiffness. Also, the burnt weld reduced the effective weld length and prompted increase in stiffness on the ideal weld considered in FE.

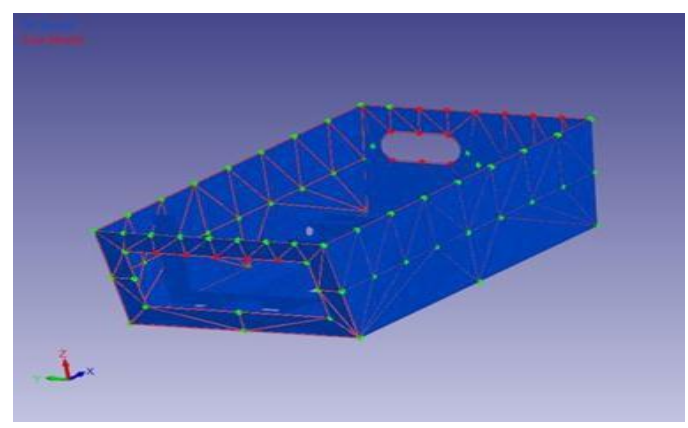

Figure 7: Node point pair with 67 points

On the other hand, the 17 points which were showing high displacements and localized flexing in EMA (shown in Figure 5) due to reduced stiffness of the actual model at these locations were disabled as sub components were not modeled with varying stiffness in FE and hence correlation was carried out with the remaining 67 points with the optimized model. The corresponding node point pair is as shown in the Figure 7.

\subsection{Mode shape pair and MAC after optimization}

The results of the mode shape pairing for the optimized model with 67 node point is as shown in Table 3. The corresponding MAC matrix is as shown Figure 8. 
Table 3: Mode shape pairs of the model after optimization

\begin{tabular}{||c|c|c|c|c|c|c|}
\hline PAIR & FEA & $\begin{array}{c}\text { FREQ. } \\
(\mathrm{Hz})\end{array}$ & EMA & $\begin{array}{c}\text { FREQ. } \\
(\mathrm{Hz})\end{array}$ & $\begin{array}{c}\text { Diff. } \\
(\%)\end{array}$ & $\begin{array}{c}\text { MAC } \\
(\%)\end{array}$ \\
\hline 1 & 1 & 54.06 & 1 & 53.73 & 0.61 & 92.7 \\
\hline 2 & 2 & 67.15 & 2 & 69.45 & -3.31 & 69 \\
\hline 3 & 3 & 71.98 & 3 & 80.47 & -10.56 & 75.5 \\
\hline 4 & 4 & 80.14 & 4 & 83.28 & -3.77 & 42.2 \\
\hline 5 & 5 & 84.61 & 5 & 93.61 & -9.62 & 37.2 \\
\hline 6 & 6 & 133.86 & 6 & 124.91 & 7.16 & 70.4 \\
\hline
\end{tabular}

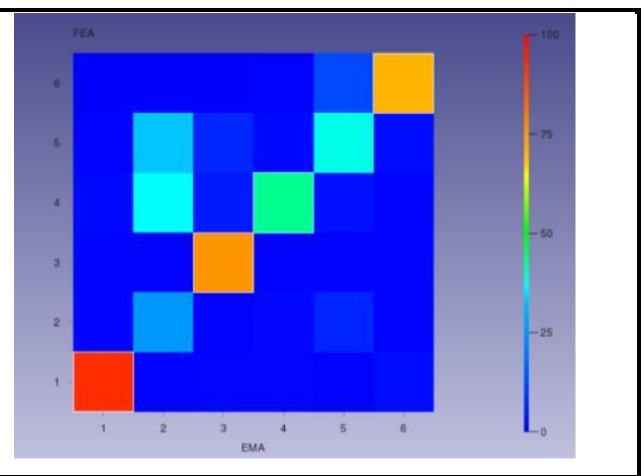

Figure 8: Graphical representation of

MAC

The frequency difference between EMA and FEA decreased significantly from the earlier $24.28 \%$ to $10.56 \%$. This is because the optimization altered the localized stiffness which was not considered earlier. The acceptance criteria of MAC were kept at $40 \%$. The modes 1, 3, 4 and 6 satisfied the criteria. The mode 5 obtained in EMA showed a slight irregularity in EMA simulation. This may be due to improper identification of residues for this mode in EMA and hence MAC of $37.2 \%$ was accepted for this mode. In order to correlate mode 2, a set of 50 points was considered which gave a MAC of $69 \%$. The plots of FE and EMA mode shape comparison for the analyzed 6 modes are as shown in Figure 9(af).

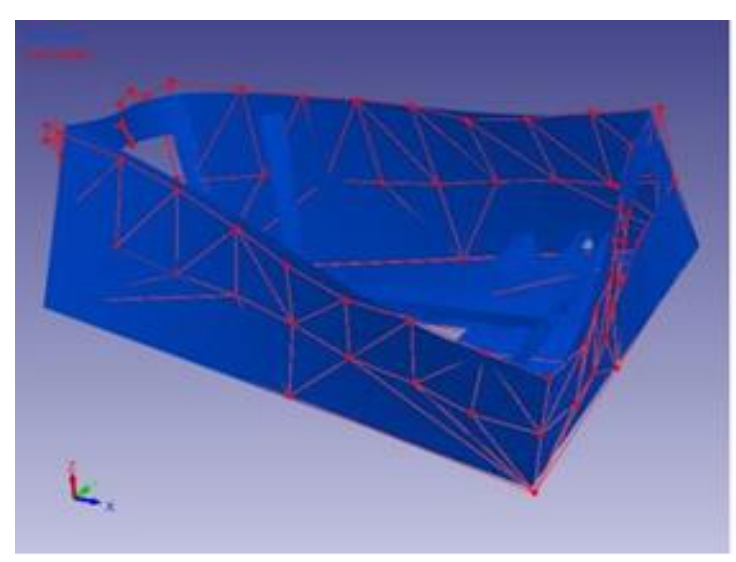

a. Mode 1

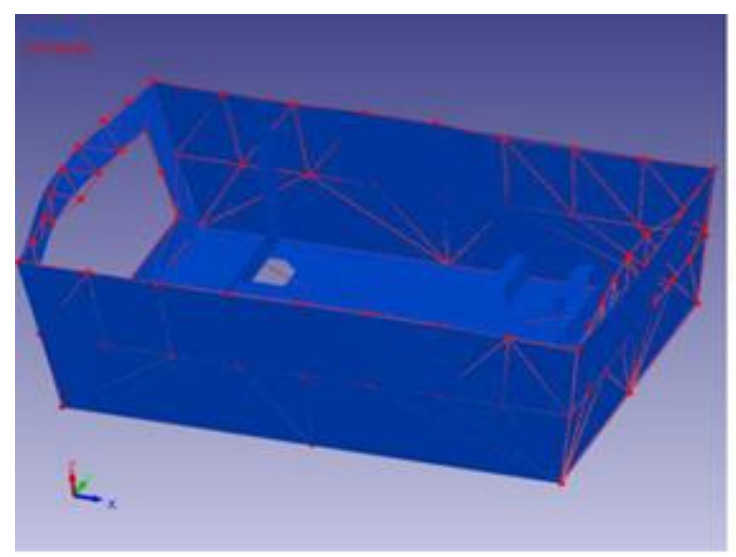

c. Mode 3

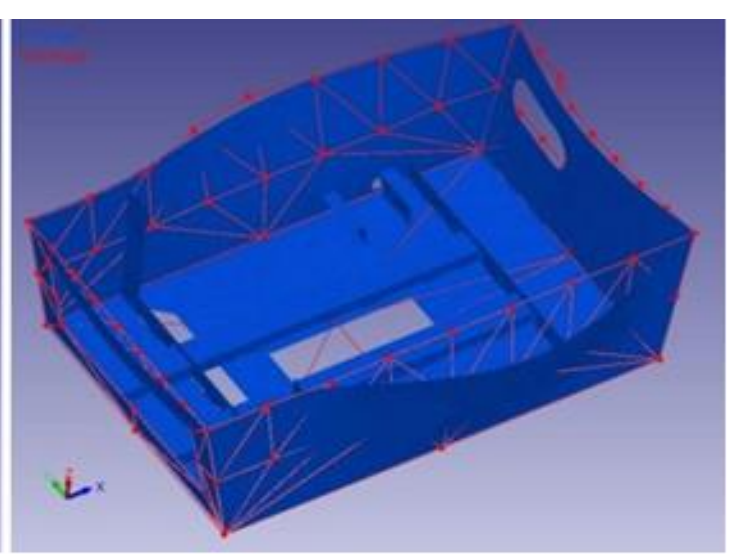

b. Mode 2

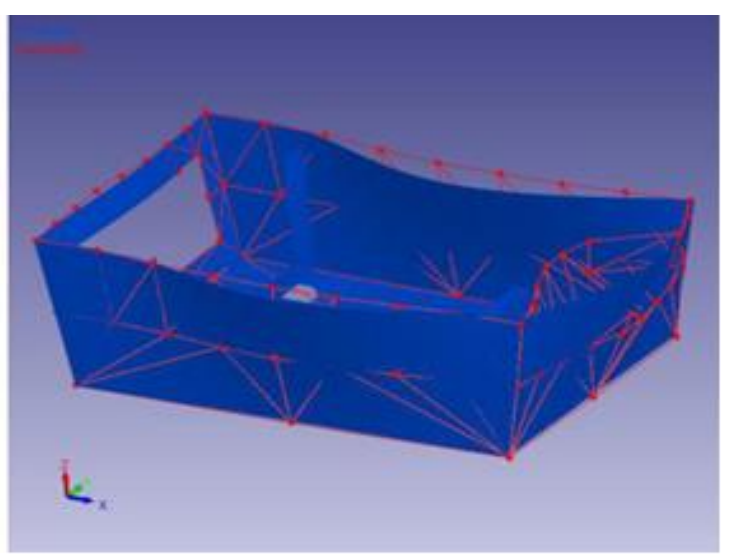

d. Mode 4 


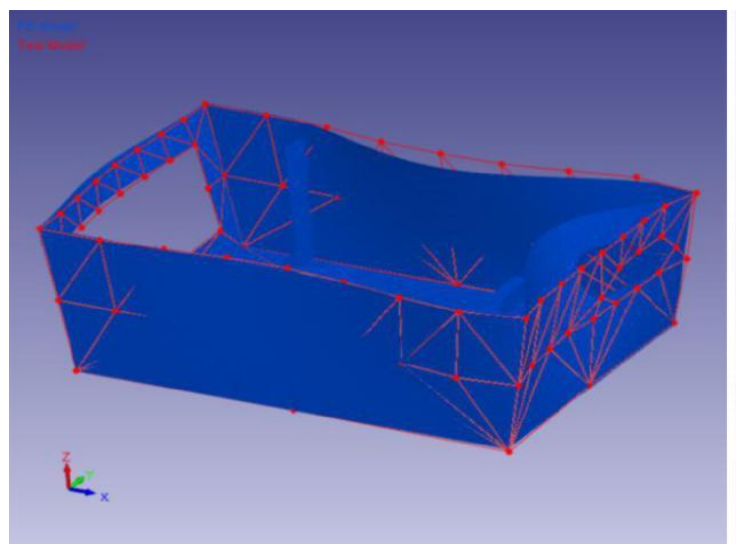

e. Mode 5

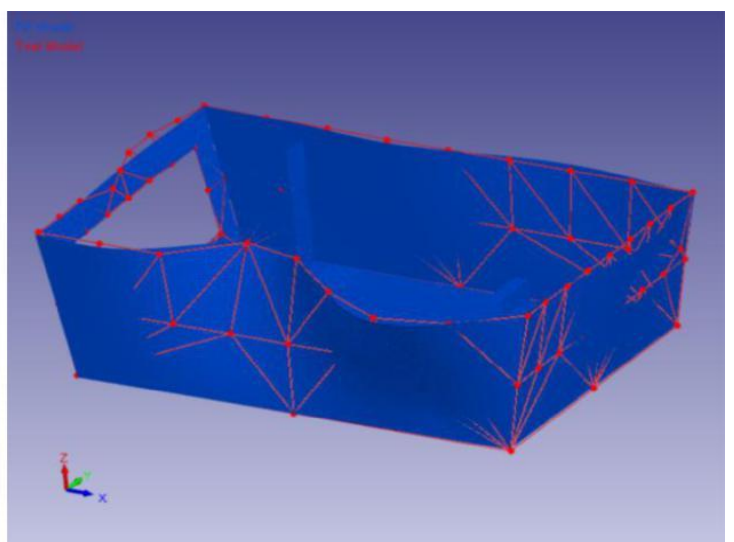

f. Mode 6

Figure 9 (a-f): FEA (in blue) and EMA (in red) Mode shape pairing

\section{Conclusions}

Thus, an asymmetric thin sheet model was manufactured and the results of modal analysis obtained from simulation and EMA were correlated and studied. From the initial FE-EMA correlation activity of the model, a maximum frequency error of $24 \%$ was observed. This was because FE modeling could not incorporate the manufacturing deviations such as efficacy of the welds, changes in material property due to changes in microstructure, dimensional tolerances of components in assembly, geometric irregularities etc. FE modeling represents the ideal conditions such as perfect joints, perfectly homogeneous and isotropic material and uniform thickness. These ideal conditions do not exist practically.

The FE-EMA frequency difference was reduced from $24 \%$ to $10 \%$ due to optimization. In order to bring down FE - EMA frequency difference, FE optimization was done by modeling each and every weld as a separate component. Thus, the mechanical properties of each of the weld components could be altered. The parameters selected for optimization were Young's modulus, Poisson's ratio and thickness.

The EMA is performed only with one sample of the experimental readings taken at 84 points (1260 FRFs) for the thin sheet metal structure considered in the study. As a future scope of this study, different thin sheet structures can be analyzed in a similar fashion and findings of this study can be verified.

\section{References}

1. Don Graff, Modal Checks for Dynamics, User manual guide, MSC Software Corporation, 2012

2. Avitabile P, Modal space (in our little world), University of Massachusetts Lowell: Structural dynamics and Acoustic systems Laboratory, Rev 072014, 2014

3. Brüel \& Kjær, Experimental Modal Analysis, Brüel \& Kjær Sound\& Vibration Measurement A/S, 2003

4. Fatih Altunel, Mehmet Celik, Mehmet Calişkan, A Correlation Improvement Technique for Model Updating of Structures, International Journal of Structural Stability and Dynamics, Vol. 16 (2016) 1550049, 2016

5. Peter Pavelka, Róbert Huňady, Martin Hagara, Result Correlation of Experimental and Numerical Modal Analysis of a Steel Beam, American Journal of Mechanical Engineering, 2016, Vol. 4, No. 7, pp 376-379

6. Koushik. S, Static and Vibration Analysis of Engine Mounting Bracket of TMX 20-2 using OptiStruct, Altair Technology conference, 2013

7. John E Mottershead, Michael I Friswell, Model Updating of Joints and Connections, International Conference on Structural Dynamics Modelling, 2002, p. 11 
8. Matteo Palmonella, Michael I. Friswell, John E. Mottershead, Arthur W. Lees, Finite element models of spot welds in structural dynamics: review and updating, Computers and Structures, 2005, 83, pp. 648-661

9. Abdullah N A Z, Sani M S M, Rahman M M, Zaman I, Correlation of numerical and experimental analysis for dynamic behaviour of a body in white (BIW) structure, MATEC Web of Conferences, 2017, issue 10.51/matecconf/20179001020

10. C. Schedlinski , F. Wagner, K. Bohnert, J. Frappier, A. Irrgang, R. Lehmann, A. Müller, Test-Based Computational Model Updating of a Car Body in White, Journal of Sound and Vibration, 2005, pp. 19-23

11. Rani M A, Aminudin B A, Yunus M and Ouyang H, Model Updating for a thin steel sheet welded structure, 20th International Congress on sound and Vibration, 2013, pages 9 